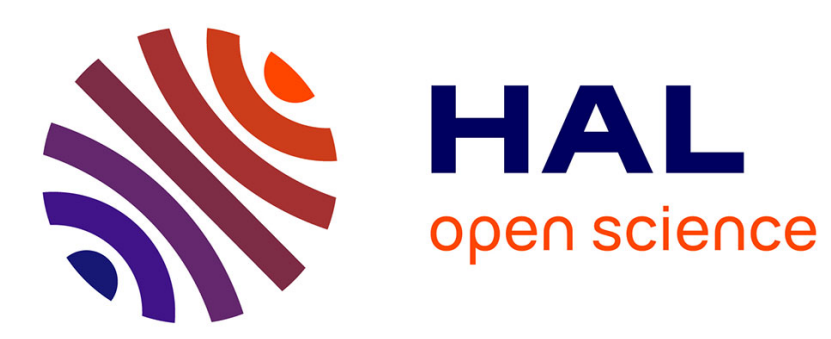

\title{
Liénard systems and potential-Hamiltonian decomposition I - Methodology
}

Jacques Demongeot, Nicolas Glade, Loïc Forest

\section{To cite this version:}

Jacques Demongeot, Nicolas Glade, Loïc Forest. Liénard systems and potential-Hamiltonian decomposition I - Methodology. Comptes rendus de l'Académie des sciences. Série I, Mathématique, 2007, 344, pp 121-126. hal-00211816

\section{HAL Id: hal-00211816 https://hal.science/hal-00211816}

Submitted on 23 Jan 2008

HAL is a multi-disciplinary open access archive for the deposit and dissemination of scientific research documents, whether they are published or not. The documents may come from teaching and research institutions in France or abroad, or from public or private research centers.
L'archive ouverte pluridisciplinaire HAL, est destinée au dépôt et à la diffusion de documents scientifiques de niveau recherche, publiés ou non, émanant des établissements d'enseignement et de recherche français ou étrangers, des laboratoires publics ou privés. 


\title{
Systèmes de Liénard et décomposition potentielle-Hamiltonienne I - Méthodologie
}

\section{Liénard systems and potential-Hamiltonian decomposition I - Methodology}

\author{
a Institut Universitaire de France \\ b TIMC-IMAG UMR CNRS 5525 \\ University J. Fourier Grenoble \\ Faculty of Medicine 38700 La Tronche France \\ Tel. 0456520108 \\ FAX 0476768844
}

Jacques DEMONGEOT ${ }^{\mathrm{a}, \mathrm{b}}$ Nicolas GLADE ${ }^{\mathrm{b}}$ Loic FOREST $^{\mathrm{b}}$

\begin{abstract}
Following the Hodge decomposition of regular vector fields we can decompose the second member of any Liénard system into 2 (non unique) polynomials, first corresponding to potential and second to Hamiltonian dynamics. This polynomial Hodge decomposition is called potential-Hamiltonian, denoted PH-decomposition, and we give it for any polynomial differential system of dimension 2. We will give in a further Note an algorithm expliciting the PH-decomposition in the neighborhood of particular orbits like a limit-cycle for Liénard systems, the method being applicable for any polynomial differential system of dimension 2 .
\end{abstract}

\section{Résumé}

Un système de Liénard est un système différentiel du second ordre, défini sur $\Re^{2}$, du type : $d x / d t=y, d y / d t=$ $-g(x)+y f(x)$ où $g$ et $f$ sont des polynômes. Un tel système est susceptible dêtre décomposé, de manière non unique, en 2 parties polynomiales, l'une potentielle et l'autre hamiltonienne, c'est-à-dire qu'il existe deux polynômes $P$ et $H$, définis sur $\Re^{2}$ à valeurs dans $\Re$, vérifiant $d x / d t=-\partial P / \partial x+\partial H / \partial y, d y / d t=-\partial P / \partial y-\partial H / \partial x$.

On montre, en utilisant la décomposition de Hodge des champs de vecteurs réguliers, que le second membre d'un tel système est décomposable en 2 polynômes, l'un correspondant à une dynamique de gradient et l'autre à une dynamique hamiltonienne. Cette décomposition de Hodge polynomiale est appelée potentielle-hamiltonienne, notée $\mathrm{PH}$-décomposition, et nous en donnons la formule pour tout système différentiel polynomial du plan. Nous donnerons, dans une Note ultérieure, un algorithme permettant d'obtenir une formule explicite de la PH-décomposition au voisinage d'orbites particulières, telles qu'un cycle limite dans le cas des systèmes de Liénard, la méthode étant applicable à tout système différentiel polynomial du plan.

\section{Version abrégée}

Les systèmes de Liénard sont des Equations Différentielles Ordinaires de Dimension 2 (2D-EDO) du type : $d x / d t=y, d y / d t=-g(x)+y f(x)$, où $f$ et $g$ sont des polynômes.

Le système de van der Pol (cf. Figure 1) en est un bon exemple [2-4], utilisé pour modéliser de nombreux systèmes biologiques régulés, dont le système cardiaque. Les polynômes $g$ et $f$ d'un système de van der Pol

Email addresses: jacques.demongeot@imag.fr (Jacques DEMONGEOT), nicolas.glade@imag.fr (Nicolas GLADE), loic.forest@imag.fr (Loic FOREST). 
sont définis par : $g(x)=x$ et $f(x)=\mu\left(1-x^{2}\right)$

L'unique cycle-limite d'un système de van der Pol, lorsqu'il existe, est une courbe non algébrique difficile à approximer et il peut être très utile, dans les applications, d'en avoir une estimation polynomiale. Nous proposons donc, de manière générale, une décomposition des EDO régulières, dite potentielle-hamiltonienne, dont l'existence est fondée théoriquement sur la décomposition de Hodge [5-8].

Une ODE est dite potentiel-hamiltonienne décomposable, s'il existe un couple de polynômes $(P, H)$, tel que: $d x / d t=-\partial P / \partial x+\partial H / \partial y, d y / d t=-\partial P / \partial y-\partial H / \partial x$

La partie du flot de vecteurs vitesse définie par $P$ correspond à une dynamique de descente de plus fort gradient sur la surface représentative de $P$ et la partie définie par $H$ correspond à une dynamique sur les courbes de niveau de la surface représentative de $H$.

Un exemple de système hamiltonien pur classique est celui du système de Lotka-Volterra, utilisé pour modéliser les interactions proie/prédateur [9]. Des exemples de systèmes différentiels purs potentiels sont les systèmes de type n-switch, à second membre défini par une cinétique de Hill compétitive (équation 4), pour lesquels on peut obtenir explicitement la formule de définition de $P$ et donc tracer la surface correspondante et en localiser les minima, qui sont les états stationnaires du n-switch (cf. Figure 3). Un exemple de système polynomial mixte potentiel-hamiltonien est le système chimique de Lotka, pour lequel il est facile d'exhiber $P$ et $H[22]$.

Pour généraliser la décomposition ci-dessus, on montre un théorème donnant une formule générale de décomposition potentielle-hamiltonienne (non unique), pour des ODE du type : $d x / d t=f(x, y), d y / d t=$ $g(x, y)$, où $f$ et $g$ sont des polynômes. A partir de cette formule générale, nous donnerons, dans deux prochaines notes, un algorithme permettant d'obtenir la décomposition potentielle-hamiltonienne dans le cas des systèmes de Liénard, puis de l'appliquer à des problèmes biologiques, dans lesquels il existe un grand intérêt à décomposer le flot en une partie potentielle (dont les paramètres sont responsables d'une modulation de fréquence d'un signal biologique, dans le cas d'une trajectoire du système de type cycle-limite) et une partie hamiltonienne (dont les paramètres sont responsables d'une modulation d'amplitude).

\section{Introduction}

Liénard systems [1] are 2-dimensional ordinary differential equations (2D ODEs) defined by $d x / d t=y$, $d y / d t=-g(x)+y f(x)$, where $g$ and $f$ are polynomials. The use of Liénard systems is universal in biological modeling, especially for physiological processes. A simple example of a Liénard system is given by the van der Pol system used to model the cardiac and respiratory rhythms [2-4], where :

$$
g(x)=x, \quad f(x)=\mu\left(1-x^{2}\right)
$$

For $\mu>0$, it presents a limit-cycle bifurcating from the stationary state located at the origin, which becomes progressively (as the anharmonic parameter $\mu$ increases) very far from a circle, and reciprocally becomes circular when $\mu$ tends to 0 (fig. 1).
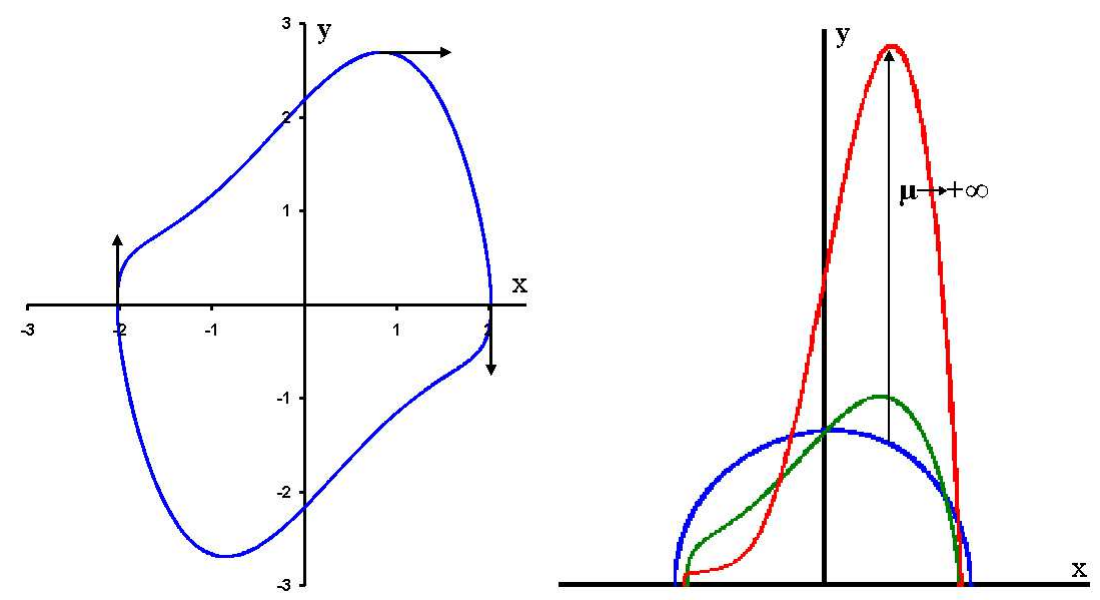

Figure 1. - van der Pol system ( $\mu=1$, left) and $\mu$ tending to infinity in the upper phase plane (right) 
For many applications, it is essential to approximate the limit-cycle (which is a non-algebraic closed curve of the plane) by a polynomial closed curve. A manner to do it is to decompose the second member of the van der Pol differential system into 2 polynomial parts, a potential one and a Hamiltonian one, contour lines of the Hamiltonian part being good candidates for estimating the limit-cycle.

\section{Potential-Hamiltonian decomposition and Hodge decomposition}

A 2D ODE $d x / d t=f(x, y), d y / d t=g(x, y)$ is called potential-Hamiltonian decomposable (PH-decomposable), if and only if there is at least one couple of polynomials $(\mathrm{P}, \mathrm{H})$ such as:

$$
d x / d t=-\partial P / \partial x+\partial H / \partial y, \quad d y / d t=-\partial P / \partial y-\partial H / \partial x
$$

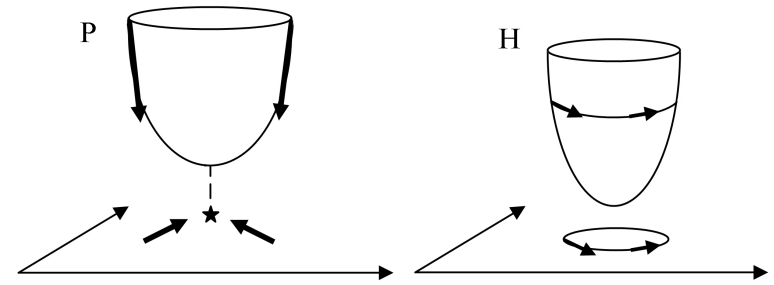

Figure 2. - Schematic representation of the gradient (potential P) flow (left) and of the conservative (Hamiltonian H) flow (right)

The potential flow is the projection on the $2 \mathrm{D}$ phase plane of a steepest descent velocity vector field on the surface defined by the potential $\mathrm{P}$, and the Hamiltonian flow the vector field tangential to contour lines of the surface generated by $H$ (fig. 2). The Hodge decomposition [5-8] ensures the existence of regular $P$ and $H$ functions (e.g. $L^{2}$ or $C^{1}$ ) for sufficiently smooth differential systems, e.g. ODEs having a second member of the same level of regularity $L^{2}$ or $C^{1}$. We try here to obtain explicit expressions (if possible polynomials) for $P$ and $H$. As example, we have the Lotka-Volterra system, a pure Hamiltonian system, introduced by V. Volterra to account for the fluctuations observed in the struggle for life between a prey population of size $x$ and a predator population of size $y[9]$ :

$$
\begin{aligned}
& d x / d t=x(a-b y), \quad d X / d t=\partial H / \partial Y \\
& d y / d t=y(c x-d), \quad d Y / d t=-\partial H / \partial X, \quad \text { with } H(X, Y)=-c e^{X}+d X-b e^{Y}+a Y
\end{aligned}
$$

by changing variables $X=\log (x)$ and $Y=\log (y)$ (population 'affinities'). The trajectories are just contour lines of $\mathrm{H}$.

\section{A pure potential system, the n-switch model}

A n-switch model is used to formalize fully connected interaction networks with only inhibitory interactions. Such a system has been proposed in [10] to model genetic and metabolic regulations and is encountered in plant growth [11-13], embryogenesis [14,15] and neural networks [16,18], in which hormones (like auxine), proteins (like transduction peptide) or neuro-transmitters (like Gaba) secreted by a part of the system (e.g. apex in plants) inhibit the growth or activity of the other parts (e.g. cotyledonary buds in plants) (fig. 3). We can represent each inhibition by a Hill competition with a decay rate $\nu$, constant entry coefficients $\alpha_{i}$ 's, a cooperativity coefficient $c$, a maximal reaction rate $\sigma$ and affinity coefficients $a_{i}$ 's as in the following system of equations, where $x_{i}$ denotes the concentration (or the size, or the amount) of the component $X_{i}$ in inhibitory interaction with all other components of the n-switch system [10]:

$$
\forall i=1 . . n, \quad \frac{d x_{i}}{d t}=-\nu x_{i}+\frac{\sigma a_{i} x_{i}^{c}}{1+\sum_{j=1}^{n} a_{j} x_{j}^{c}}+\alpha_{i}
$$

If we consider a n-switch with $c=1$ and $\forall i=1 . . n, \alpha_{i}=0, a_{i}=1: d x_{i} / d t=-\nu x_{i}+\sigma x_{i} /\left(1+\sum_{j=1}^{n} x_{j}\right)$, with $\sigma>\nu$, then the non-zero stationary states have $m \geq 1$ components $x_{i}^{*}$ equal to $(\sigma-\nu) / m \nu>0$ and the others equal to 0 . The Jacobian matrix in these stationary states has only one non-zero eigenvalue $\lambda=\nu(\nu-\sigma) / \sigma<0$ and we need a complete stability analysis done in [10] in the general case. Studying the 
stability basins topography is easier if we decompose the flow. By changing variables $y_{i}=\left(x_{i}\right)^{1 / 2}$ we see that the system is purely gradient with : $P(y)=\nu \sum_{i} y_{i}^{2} / 4-\sigma \log \left(1+\sum_{i} a_{i} y_{i}^{2 c}\right) / 4 c-\sum_{i} \alpha_{i} \log y_{i} / 2$ (cf. in fig. 3 a 2 -switch with 3 stable stationary states). Replacing Hill competition by an allosteric inhibition, the system remains pure potential (cf. $[19,20]$ for a first proof of this result):

$d x_{i} / d t=-\nu x_{i}+\sigma x_{i}\left(\left(1+x_{i}\right)^{c-1} \prod_{j \neq i}\left(1+x_{j}\right)^{c}+a_{i}\left(1+a_{i} x_{i}\right)^{c-1} \prod_{j \neq i}\left(1+a_{j} x_{j}\right)^{c}\right) /\left(\prod_{k=1}^{n}\left(1+x_{k}\right)^{c}+\prod_{k=1}^{n}(1+\right.$ $\left.\left.a_{k} x_{k}\right)^{c}\right)+\alpha_{i}$.

Hence, because $d y_{i} / d t=1 /\left(2 y_{i}\right) d x_{i} / d t$, we have $d y_{i} / d t=-\partial P / \partial y_{i}$, with:

$P(y)=\nu \sum_{i=1}^{n} y_{i}^{2} / 4-\sigma \log \left(\prod_{i=1}^{n}\left(1+y_{i}^{2}\right)^{c}+\prod_{i=1}^{n}\left(1+a_{i} y_{i}^{2}\right)^{c}\right) / 4 c-\sum_{i=1}^{n} \alpha_{i} \log y_{i} / 2$, where $y_{i}=\left(x_{i}\right)^{1 / 2}$.
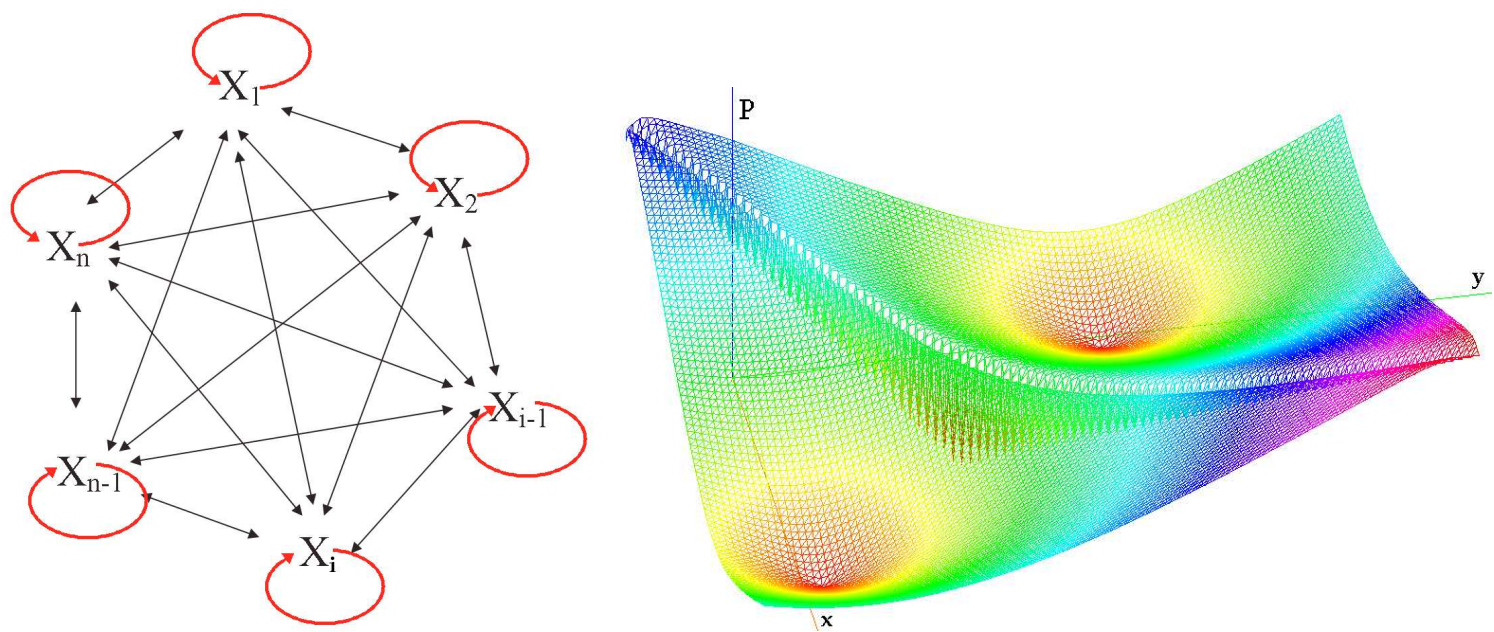

Figure 3. - Fully connected inhibitory interactions (except eventually auto-loops) of a n-switch (left) and potential $\mathrm{P}$, if $\mathrm{n}=2$ (right).

We represent on Figure 3 (right) the surface of the potential $P$ for a 2 -switch $\left(\nu=1, \mathrm{c}=2, \sigma=2, a_{i}=0.1\right.$, $\alpha=1)$ over the square $[-5,35] \mathrm{x}[-5,35]$. The local minima are located in $(1.24,24),(24,1.24)$, and $(12.35,12.35)$ on the bissectrix.

\section{Application to mixed potential-Hamiltonian systems, the Lotka and the Liénard equations}

Chemical dynamical systems frequently offer equations susceptible to be PH-decomposed [21]. A historical example is the Lotka system [22] built to model a bi-reactant oscillatory reaction:

$$
d x / d t=-x y-K x-L y, \quad d y / d t=x y+L y
$$

The decomposition is obtained by choosing

$P(x, y)=(K+y) x^{2} / 2-L y^{2} / 2-x^{3} / 6$ and $H(x, y)=-y x^{2} / 2-L y^{2} / 2-x^{3} / 6$

An other simple non-linear system is the Lorenz pendulum [23]:

$$
d x / d t=y, \quad d y / d t=-a x-x^{3}
$$

This system is pure Hamiltonian with $H(x, y)=y^{2} / 2+a x^{2} / 2+x^{4} / 4$. More generally, Liénard systems are PH-decomposable, if $h$ and $l$ are polynomials of order respectively $m$ and $p$, and if $h^{(k)}$ denotes the $k^{t h}$ derivative of $h$, we have:

$$
\begin{aligned}
P(x, y)= & \sum_{k=1}^{n}(-1)^{k} f^{(2 k-2)} y^{2 k} /(2 k) !+\sum_{k=1}^{m}(-1)^{k} h^{(2 k-2)} y^{2 k-1} /(2 k-1) !+\sum_{k=1}^{p}(-1)^{k} l^{(2 k-2)} x^{2 k-2} /(2 k-2) ! \\
H(x, y)= & \int(g+h)+y^{2} / 2+\sum_{k=1}^{n}(-1)^{k} f^{(2 k-1)} y^{2 k+1} /(2 k+1) ! \\
& +\sum_{k=1}^{m}(-1)^{k} h^{(2 k-1)} y^{2 k} /(2 k) !+\sum_{k=1}^{p}(-1)^{k+1} l^{(2 k-1)} x^{2 k-1} /(2 k-1) !
\end{aligned}
$$

Proof is just the consequence of the following Proposition, Lemmas and Theorem, available for any 2D 
ODE with sufficiently regular second member.

Proposition 1 Let's consider $V=f(x, y) \partial . / \partial x+g(x, y) \partial . / \partial y$ being a polynomial vector field, then there is a (non unique) PH-decomposition of $V$ into two parts, a potential part, which is the gradient of a polynomial $P$ and a conservative part, which is the Hamiltonian of a polynomial $H$ such that: $V=-g r a d P+h a m H$

Proof 1 Calculate div $V=D(x, y)$ and use complex coordinates $z=x+i y, \bar{z}=x-i y$. Then there is a (non unique) polynomial $P$ such that $\partial^{2} P / \partial z \partial \bar{z}=\partial^{2} P / \partial x^{2}+\partial^{2} P / \partial y^{2}=-D(x, y)$. The divergence $V+$ gradP equals 0 . Hence this divergence is a Hamiltonian polynomial vector field deriving from a Hamiltonian function $H: V+\operatorname{gradP}=(\partial H / \partial y,-\partial H / \partial x)=h a m H$

Lemma 1 Let's consider the 2D ODE : dx/dt=0, dy/dt=0 then its PH-decomposition can be any kernel of the type $P(x, y)=(f+i g)(x+i y)+(f-i g)(x-i y), H(x, y)=-i(f+i g)(x+i y)+i(f-i g)(x-i y), f$ and $g$ being differentiable functions.

Proof 2 It's sufficient to check that: $-\partial P / \partial x+\partial H / \partial y=0$ and $-\partial P / \partial y-\partial H / \partial x=0$

Lemma 2 Let's consider the 2D ODE:

$$
d x / d t=f_{1}(x, y)+f_{2}(x, y), \quad d y / d t=g_{1}(x, y)+g_{2}(x, y)
$$

where the functions $f_{1}, f_{2}, g_{1}, g_{2}$ are infinitely differentiable and integrable. If $f^{[n](m)}(x, y)$ represents the function obtained from $f$ by integrating $n$ times in $x$ and derivating $m$ times in $y$ then, for any integer $k \geq 4$ , the potential $P$ and the Hamiltonian $H$ defined by :

$P=\sum_{j \leq k, j=4 p+1}-f_{1}^{[j](j-1)}-g_{1}^{(j-1)[j]}+\sum_{j \leq k, j=4 p+3} f_{1}^{[j](j-1)} \pm g_{1}^{(j-1)[j]}+\sum_{j \leq k, j=4 p+4}^{j(j-1)[j]}-g_{2}^{[j](j-1)}+$
$\sum_{j \leq k, j=4 p+1} f_{2}^{(j-1)[j]}-g_{2}^{[j](j-1)}+\sum_{j \leq k, j=4 p+2} f_{1}^{[j](j-1)}-g_{1}^{(j-1)[j]}+$
$\sum_{j \leq k, j=4 p+3}-f_{2}^{(j-1)[j]}+g_{2}^{[j](j-1)}+\sum_{j \leq k, j=4 p+4}^{[j-1)}-f_{1}^{[j](j-1)}+g_{1}^{(j-1)[j]}$

constitute the PH-decomposition of the following ODE's:

for $k=4 p+1, d x / d t=f_{1}+f_{2}+g_{1}^{(k)[k]}-g_{2}^{[k](k)}, d y / d t=g_{1}+g_{2}+f_{1}^{[k](k)}-f_{2}^{(k)[k]}$ for $k=4 p+2, d x / d t=f_{1}+f_{2}+f_{1}^{[k](k)}+f_{2}^{(k)[k]}, d y / d t=g_{1}+g_{2}+g_{1}^{(k)[k]}+g_{2}^{[k](k)}$ for $k=4 p+3, d x / d t=f_{1}+f_{2}-g_{1}^{(k)[k]}+g_{2}^{[k](k)}, d y / d t=g_{1}+g_{2}-f_{1}^{[k](k)}+f_{2}^{(k)[k]}$ for $k=4 p+4, d x / d t=f_{1}+f_{2}-f_{1}^{[k](k)}-f_{2}^{(k)[k]}, d y / d t=g_{1}+g_{2}-g_{1}^{(k)[k]}-g_{2}^{[k](k)}$

If $f_{1}, f_{2}, g_{1}, g_{2}$ are polynomials of order less than $n$, then all these ODE's are equal to (7), since $k>n$.

Proof 3 It's sufficient to check that: $d x / d t=-\partial P / \partial x+\partial H / \partial y, d y / d t=-\partial P / \partial y-\partial H / \partial x$ and $f_{1}^{[k](k)}=$ $f_{2}^{(k)[k]}=g_{1}^{(k)[k]}=g_{2}^{[k](k)}=0, k>n$

Theorem 1 Let's consider the 2D ODE: $d x / d t=f(x, y), d y / d t=g(x, y)$ where $f$ and $g$ are polynomials: $f(x, y)=\sum_{i \geq j} a_{i j} x^{i} y^{j}+\sum_{i<j} c_{i j} x^{i} y^{j}$ and $g(x, y)=\sum_{i \geq j} b_{i j} x^{i} y^{j}+\sum_{i<j} d_{i j} x^{i} y^{j}$

Then the most general PH-decomposition is obtained by choosing

$\sum_{j \leq k, j=4 p+1}-f_{1}^{[j](j-1)}-g_{1}^{(j-1)[j]}+\sum_{j \leq k, j=4 p+3} f_{1}^{[j](j-1)}+g_{1}^{(j-1)[j]}+\sum_{j \leq k, j=4 p+2}-f_{2}^{(j-1)[j]}-g_{2}^{[j](j-1)}+$
$\sum_{j=4 p+4}^{(j-1)[j]}+g_{2}^{[j](j-1)}+(f+i g)(x+i y)+(f-i g)(x-i y)$ 


$$
\begin{gathered}
\sum_{j \leq k, j=4 p+1} f_{2}^{(j-1)[j]}-g_{2}^{[j](j-1)}+\sum_{j \leq k, j=4 p+2} f_{1}^{[j](j-1)}-g_{1}^{(j-1)[j]}+ \\
\sum_{j \leq k p+3}-f_{2}^{(j-1)[j]}+g_{2}^{[j](j-1)}+\sum_{j \leq k, j=4 p+4}^{[j](j-1)}+g_{1}^{(j-1)[j]}-i(f+i g)(x+i y)+i(f-i g)(x-i y)
\end{gathered}
$$

where $f, g$ are any differentiable functions and where:

$$
\begin{aligned}
& f_{1}=\sum_{i>j} a_{i j} x^{i} y^{j}+\frac{1}{2} \sum_{i=j} a_{i j} x^{i} y^{j}, \quad f_{2}=\sum_{i<j} c_{i j} x^{i} y^{j}+\frac{1}{2} \sum_{i=j} a_{i j} x^{i} y^{j} \\
& g_{1}=\sum_{i>j} b_{i j} x^{i} y^{j}+\frac{1}{2} \sum_{i=j} b_{i j} x^{i} y^{j}, \quad g_{2}=\sum_{i<j} d_{i j} x^{i} y^{j}+\frac{1}{2} \sum_{i=j} b_{i j} x^{i} y^{j}
\end{aligned}
$$

Proof 4 The existence of a PH-decomposition is ensured by the above Proposition. Then to obtain P and $H$, it's sufficient to apply the above Lemmas 1 and 2

\section{Conclusion}

In this article, we have shown the possibility to decompose any sufficiently regular 2D-differential system into two parts : a potential one having a dissipative gradient-like behaviour responsible, in general, for the amplitude of a periodic signal generated by the system, and another one, Hamiltonian, expressing the conservative part of the flow responsible for the frequency of this periodic signal. This decomposition, called PH-decomposition if the potential $P$ and the Hamiltonian $H$ are polynomials, is non unique, but we easily conceive that we can estimate the coefficients of $P$ and $H$ by obliging the dynamical flow to pass through certain known points on orbits of the studied system. Then we have obtained a way to algebraically estimate these orbits. The algorithm for doing this approximation (in particular in the case of limit-cycles) will be given in a further Note.

\section{Acknowledgements}

We are very indebted to R. Thom (died in October 2002, formerly President of the French Society for Theoretical Biology) for opening the new field of modeling biological systems with a qualitative approach using singularity theory, and to J.P. Françoise for stimulating discussions and helpful suggestions.

\section{References}

[1] Liénard A., Etude des oscillations entretenues, Revue Générale de l’Electricité 23 (1928) 901-912 \& 946-954

[2] van der Pol B., van der Mark J., The heartbeat considered as a relaxation oscillation and an electrical model of the heart, Philos. Mag. 6 (1928) 763-775.

[3] van der Pol B., van der Mark J., Le battement du cœur considéré comme oscillation de relaxation et un modèle électrique du cœur, Onde élec. (1928) 365-392.

[4] Pham Dinh T., Demongeot J., Baconnier P., Benchetrit G., Simulation of a biological oscillator: the respiratory rhythm, J. Theoret. Biol. 103 (1983) 113-132.

[5] Iwaniec T., Scott C., Stroãolini B., Nonlinear Hodge theory on manifolds with boundary, Annali Mat. Pura \& Appl. 177 (1999) $37-115$

[6] El Kacimi-Alaoui A., Hector G., Basic Hodge decomposition for a Riemannian foliation, Ann. Institut Fourier 36 (1986) 207-227.

[7] Collela P., Trebovich D.P., Numerical simulation of incompressible viscous flow in deforming domains, Proc. Natl. Acad. Sci. USA 96 (1999) 5378-5381.

[8] Nazarenko A., Canonical Realization of the Poincaré Algebra for a Relativistic System of Charged Particles Plus Electromagnetic Field, Proceedings of Institute of Mathematics of NAS of Ukraine 30 (2000) 343-349.

[9] Volterra V., Fluctuations dans la lutte pour la vie, leurs lois fondamentales et de réciprocité, Bull. SMF 67 (1939) $135-151$.

[10] Cinquin O., Demongeot J., High-dimensional switches and the modeling of cellular dfferentiation, J. Theor. Biol. 233 (2005) 391-411.

[11] Demongeot J., Thomas R., Thellier M. A mathematical model for storage and recall functions in plants. C.R. Acad. Sc., Sciences de la Vie 323 (2000) 93-97. 
[12] Demongeot J., Aracena J., Thuderoz F., Baum T.P., Cohen O., Genetic regulation networks: circuits, regulons and attractors, C. R. Acad. Sci. Biologies 326 (2003) 171-188.

[13] Thellier M., Demongeot J., Guespin J., Ripoll C., Norris V., Thomas R., A logical (discrete) formulation model for the storage and recall of environmental signals in plants, Plant Biol. 10 (2004) 1055-1075.

[14] Jolliot A., Prochiantz A., Transduction peptides: from technology to physiology, Nature Cell Biology 6 (2004) 189-196.

[15] Isalan M., Lemerle C., Serrano L, Engineering gene networks to emulate Drosophila embryonic pattern formation, Plos Biology 3 (2005) 488-496.

[16] Wang X.J., Rinzel J., Alternating and synchronous rhythms in reciprocally inhibitory model neurons, Neural Computation 4 (1992) 44-97.

[17] Skinner F.K., Kopell N., Marder E., Mechanisms for oscillation and frequency control in reciprocally inhibitory model neural networks, Journal of Computational Neuroscience 1 (1994) 69-87.

[18] Taylor A.L., Cottrell G.W., Kristan W.B., Analysis of oscillations in a reciprocally inhibitory network with synaptic depression, Neural Computation 14 (2002) 561-581.

[19] Demongeot J., Theoretical study of attractors in enzymatic systems, in: Paulré B.E. (Ed.), System dynamics and analysis of change, Paulré B.E. ed., North Holland, Amsterdam, 1981, pp. 102-122.

[20] Demongeot J., Existence de solutions périodiques pour une classe de systèmes différentiels gouvernant la cinétique de chaînes enzymatiques oscillantes, Lecture Notes in Biomaths 41 (1981) 40-62.

[21] Quapp W., Reaction Pathways and Projection Operators: Application to String Methods, J. Comput. Chem. 25 (2004) $1277-1285$.

[22] Lotka A.J., Contribution to the theory of periodic reactions, J. Phys. Chem. 14 (1910) 271-279.

[23] Clerc M., Coullet P., Tirapegui E., The stationary instability in quasi-reversible systems and the Lorenz pendulum, International Journal of Bifurcation and Chaos 11 (2001) 591-603. 